\title{
Models for the Optimization and Risk Management of Energy Conversion Networks
}

\author{
Jeffrey Kantor and Patrick Mousaw
}

\begin{abstract}
Using first and second law principles from finitetime thermodynamics, this paper introduces a class of bilinear models suitable for the optimization and risk management of commodity energy conversion processes. The model is intended for use at a high level to predict the efficiency of energy conversion in campus scale utilities with complex energy requirements, fuel sources, and significant operational flexibility. The bilinear character of the model derives from the second law which results in a multiplicative coupling between entropy flux and temperature driving forces. The paper offers three main results:

1) Economic optimization of this model yields a non-convex bilinear optimization problem.

2) Special cases for optimal operation reduce to generalized eigenvalue problems, or more complex rank constraints that could be solved using numerical algebraic geometry.

3) A computational strategy based on a linear outer approximation coupled with a branch and bound methodology to reduce the search region.
\end{abstract}

\section{INTRODUCTION}

The operation of campus or municipal scale energy systems involves a complex interplay of control, optimization, and risk management in a changing and uncertain environment. An operator's challenge is to exploit flexibility within the system to meet utility demands while minimizing operating costs and maximizing the value of the real assets. For typical campus co-generation facilities, some of the flexibility is of an operational nature, such as the flexibility to use multiple fuels to produce heat, chilling, electricity, and process heat. In addition, financial flexibility is gained through the use of financial instruments, including futures and options, to mitigate risk in the commodity markets. An additional source of financial flexibility is in the design of interuptible contracts for the supply of gas or electricity.

Our current research program focuses on the development of models, techniques, and insights to assist in optimizing the economic performance of campus scale energy conversion systems. To maximize value, we are interested in integrating the operational, financial, and contractual control of energy conversion systems. Our earlier work [2], [9], and the work of others (see, for example, [6], [7], [12]), has demonstrated the value of integrating hedging and operational decisions in electricity generation. There are many open problems and opportunities for basic research.

In this paper, we consider the specific issue of developing models to support our study of optimization and risk management for energy conversion systems. We seek a class

The authors are with the Department of Chemical \& Biomolecular Engineering, 182 Fitzpatrick Hall, University of Notre Dame, Notre Dame, IN 46556 USA

Correspondence should be addressed to Kantor.1@nd.edu of models that captures the essential behaviors and tradeoffs involved in energy conversion without getting bogged down in detailed equipment descriptions. In particular, the extensive literature on finite time thermodynamics [1], [3], [4], [8] may offer such a framework. However, despite the a rich literature, relatively little known about the general nature of this class of models, nor is there a 'systems' framework that extends application to larger-scale complex utilities. Those are the issues we address in this paper.

Here we use the general approach of finite-time thermodynamics to develop a general class of bilinear models suitable for optimization studies. By incorporating first and second law thermodynamics, the model predicts the efficiency of energy conversion in campus scale utilities with complex energy requirements, fuel sources, and significant requirements for operational flexibility.

\section{Component Models}

In this section we develop simple models for key components comprising an energy utility. We decompose larger systems into basic components including heat nodes, thermal links, heat engines, and heat pumps. These basic components and associated thermodynamic equations are described below. More complex models, which we term 'energy conversion networks' (ECN), are then constructed from these basic components.

\section{A. Basic Components}

Heat Node: Heat nodes (Fig. 1) typically constitute the largest number of components in our ECN models. Each heat node is characterized by a temperature $T_{i}$ and a net external heat flux $Q_{i}$. Heat nodes are coupled by thermal links, heat engines, or heat pumps.

Inputs to heat nodes represent the energy flux or power entering the node. For a heat node connected to a heat engine, for example, the heat flux represents energy flux to or from the heat engine. If a heat node is connected to a heat pump, the heat flux represents chilling capacity. Our convention is to organize diagrams so that higher temperature nodes are generally located above lower temperature nodes.

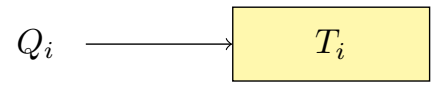

Fig. 1. 
Heat Transport and Thermal Links: Heat transport between two heat nodes is represented by a thermal link (Fig. 2). The model proposed in this paper uses Newton's law for heat transport: $Q_{i \rightarrow j}=K_{i j}\left(T_{i}-T_{j}\right)$. Other empirical descriptions of heat transport, such as the Stefan-Boltzmann law, are encountered in the literature on finite-time thermodynamics but are not explored further here.

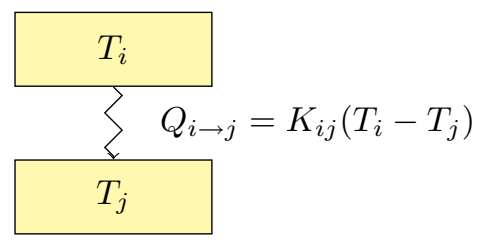

Fig. 2. Heat Transport

Heat Engines: Heat engines (Fig. 3) represent workproducing equipment. Each heat engine is characterized by an isentropic efficiency $\eta$, work flux $W$, and entropy flux $\sigma$. Each heat engine is located between two heat nodes and is assumed to operate adiabatically $\left(Q^{+}=Q^{-}+W\right)$. The equations for isentropic efficiency, entropy flux, heat fluxes, and work output for the $k^{\text {th }}$ heat engine ( $k$ indexing all heat engines and pumps) are (Eq. 1-3):

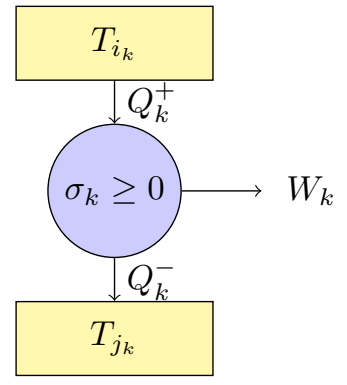

Fig. 3. Heat Engine

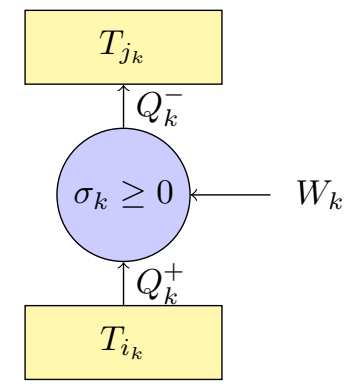

Fig. 4. Heat Pump

$$
\begin{gathered}
\eta_{k}=\frac{W_{k}}{W_{k}^{R e v}} \\
\sigma_{k}=\frac{Q_{k}^{+}}{T_{i_{k}}} \\
Q_{k}^{+}=\sigma_{k} T_{i_{k}} \\
W_{k}=\eta_{k} \sigma_{k}\left(T_{i_{k}}-T_{j_{k}}\right) \\
Q_{k}^{-}=\left(1-\eta_{k}\right) \sigma_{k} T_{i_{k}}+\eta_{k} \sigma_{k} T_{j_{k}}
\end{gathered}
$$

Heat Pumps: Heat pumps (Fig. 4) represent workconsuming equipment. Each heat pump is characterized in the same way as a heat engine, by an isentropic efficiency $\eta$, a work flux $W$, and entropy flux $\sigma$. The major difference between a heat pump and heat engine is the work flux $W$ is positive for a heat engine but negative for a heat pump. Each heat pump is also modeled between two heat nodes and assumed to be externally adiabatic. The equations for heat fluxes and work output for the $k^{\text {th }}$ heat pump are (Eq. 4-6):

$$
\eta_{k}=\frac{W_{k}^{R e v}}{W_{k}}
$$

$$
\begin{gathered}
\sigma_{k}=\frac{Q_{k}^{+}}{T_{i_{k}}} \\
Q_{k}^{+}=\sigma_{k} T_{i_{k}} \\
W_{k}=\frac{1}{\eta_{k}} \sigma_{k}\left(T_{i_{k}}-T_{j_{k}}\right) \\
Q_{k}^{-}=\left(1-\frac{1}{\eta_{k}}\right) \sigma_{k} T_{i_{k}}+\frac{1}{\eta_{k}} T_{j_{k}}
\end{gathered}
$$

Work Nodes: Work output from heat engines and work input from heat pumps are combined in work nodes to provide a cumulative work flux. Work is conserved in a work node. This conservation of work introduces an important constraint since, for example, the amount of work used by a heat pump cannot exceed the amount generated by the heat engine supplying the work. There is no specific graphical representation of work nodes in a model schematic.

\section{BILINEAR MODElS FOR ENERGy CONVERSION NETWORKS}

Next we show how the components introduced above are combined into more complex models exhibiting a characteristic bilinear coupling between temperature and entropy fluxes.

\section{A. Structure}

We introduce the following notation and structure for an energy conversion network:

- $N, M, P$ - Number of heat nodes, engines, work nodes,respectively

- $q, T$ - Temperature and heat flux vectors $(N \times 1)$

- $\sigma$ - Entropy flux vector $(M \times 1)$

- $w$ - Work flux vector $((M+P) \times 1)$

- $K$ - Thermal links matrix $(N \times N)$

- $E$ - Isentropic efficiencies matrix for heat flux calculations $(N \times N \times M)$

- $W$ - Isentropic efficiencies matrix for work flux calculations $((M+P) \times N \times M)$

- $R$ - Fuel cost vector $(N \times 1)$

- $S$ - Work value vector $((M+P) \times 1)$

With this notation and structure, equations for heat and work fluxes may be written in matrix form:

$$
\begin{gathered}
q(T, \sigma)=\left(K+\sum_{k=1}^{M} E_{k} \sigma_{k}\right) T \\
w(T, \sigma)=\left(\sum_{k=1}^{M} W_{k} \sigma_{k}\right) T
\end{gathered}
$$

The following is a summary of the matrices:

$$
\begin{aligned}
K= & {\left[\begin{array}{ccc}
K_{12} & -K_{12} & \cdots \\
-K_{12} & K_{12} & \\
\vdots & & \ddots
\end{array}\right] } \\
E_{k}= & {\left[\begin{array}{cccc}
0 & \cdots & \cdots & 0 \\
\vdots & 1 & 0 & \vdots \\
\vdots & -\left(1-\eta_{k}\right) & -\eta_{k} & \vdots \\
0 & \cdots & \cdots & 0
\end{array}\right] }
\end{aligned}
$$




$$
W_{k}=\left[\begin{array}{cccc}
0 & \eta_{k} & -\eta_{k} & 0 \\
\vdots & 0 & 0 & \vdots \\
\vdots & \vdots & \vdots & \vdots \\
0 & \eta_{k} & -\eta_{k} & 0
\end{array}\right]
$$

Matrix $K$ contains the heat transfer coefficients associated with thermal links between heat nodes. Multiple thermal links to and from a heat node may exist and the thermal conductivity constants are additive.

The matrices for $E_{k}$ and $W_{k}$ describe the coupling of heat engines within the ECN. The matrices for a heat pump have similar structure except $\frac{1}{\eta_{k}}$ replace instances of $\eta_{k}$ in both matrices. The subscripts $i$ and $j$ represent the heat nodes above and below the $k^{\text {th }}$ heat engine or pump. In the work matrix $W_{k}$, the columns with non-zero values are associated with the heat nodes above and below the heat engine or pump. The first row populated is the $k^{\text {th }}$ row and the second row populated is the $M+p^{\text {th }}$ row, where $p$ represent the work node where work is generated or consumed by the heat engine or pump.

\section{B. Optimization Statement}

An economic objective is obtained from Eq. 7 and Eq. 8 and written in terms of cost as:

$$
f(T, \sigma)=R^{\top} q-S^{\top} w
$$

This objective function is a bilinear, non-convex function since both $q$ and $w$ have the term $\sigma T$. The optimization problem is:

$$
\begin{aligned}
\min _{t, \sigma} & f=R^{\boldsymbol{\top}} q-S^{\boldsymbol{\top}} w \\
\text { s.t. } \quad & q=\left(K+\sum_{k} E_{k} \sigma_{k}\right) T \\
w & =\left(\sum_{k} W_{k} \sigma_{k}\right) T \\
T^{L} & \leq T \leq T^{U} \\
\sigma^{L} & \leq \sigma \leq \sigma^{U} \\
q^{L} & \leq q \leq q^{U} \\
w^{L} & \leq w \leq w^{U}
\end{aligned}
$$

Again, the object function $f$ represents the cost of operation. The optimization problem seeks the minimum operating cost (preferably less than zero, meaning the operation is profitable) that satisfies the constraints.

\section{Theoretical Results}

We consider here a single optimization objective

$$
J(q, T, \sigma)=\left[\begin{array}{ll}
c_{0} & d_{0}+\sum_{k=1}^{M} \sigma_{k} d_{k}
\end{array}\right]\left[\begin{array}{c}
q \\
T
\end{array}\right]
$$

were we seek first order stationary conditions such that

$$
\frac{\partial}{\partial \sigma_{k}} J(q(\sigma), T(\sigma), \sigma)=0 \quad k=1,2, \ldots, M
$$

subject to

$$
\left[\begin{array}{cc}
A & B \\
-I & K+\sum_{k=1}^{P} \sigma_{k} E_{k}
\end{array}\right]\left[\begin{array}{c}
q \\
T
\end{array}\right]=\left[\begin{array}{l}
b \\
0
\end{array}\right]
$$

In this form, the optimization problem forms a bilinear objective subject to bilinear constraints. This problem is generally nonconvex. Below we tackle this problem analytically to derive conditions for optimality, and show the nature of the resulting eigenvalue problem.

\section{A. First Order Necessary Conditions}

Expanding vectors $Q$ and $T$ in powers of $\delta_{1}, \delta_{2}, \ldots, \delta_{M}$

$$
\begin{gathered}
q(\sigma+\delta)=q_{0}(\sigma)+\sum_{k=1}^{M} \delta_{k} q_{k}(\sigma)+\cdots \\
T(\sigma+\delta)=T_{0}(\sigma)+\sum_{k=1}^{M} \delta_{k} T_{k}(\sigma)+\cdots
\end{gathered}
$$

To economize on notation, we suppress the argument $\sigma$ for the vector $T_{k}^{\prime} s$ and $q_{k}^{\prime} s$. Substituting these expansions into $J(q, T, \sigma)$, and setting first order terms in the $\sigma_{k}^{\prime} s$ to zero, we obtain the following relationships. First, for the zeroth order terms, we have a system of $2 N$ equations

$$
\begin{aligned}
A q_{0}+B T_{0} & =b \\
-q_{0}+\left(K+\sum_{k=1}^{M} \sigma_{k} E_{k}\right) T_{0} & =0
\end{aligned}
$$

Then, for the first order terms, for each $k=1,2, \ldots, P$ we have $2 N+1$ equations

$$
\begin{aligned}
A q_{k}+B T_{k} & =0 \\
E_{k} T_{0}-q_{k}+\left(K+\sum_{k=1}^{M} \sigma_{k} E_{k}\right) T_{k} & =0 \\
d_{k} T_{0}+\left(d_{0}+\sum_{k=1}^{M} \sigma_{k} d_{k}\right) T_{k} & =0
\end{aligned}
$$

Collectively, we have a system of $(2 N+1)(M+1)-1$ equations that can, in principle, be solved for the $(2 N+1)(M+$ $1)-1$ variables making up the vectors $\sigma, q_{0}, q_{1}, \ldots, q_{M}$, and $T_{0}, T_{1}, \ldots, T_{M}$.

These equations lend themselves to several useful geometrical interpretations. We are seeking values for $\sigma$ such that the constant terms lie within its range space of the linear equations formed by this system of equations. Another way to state this condition is that we seek values for $\sigma$ such that the augmented matrix for the above set of linear equations has a rank defect. This constitutes a generalized eigenvalue 
problems in the elements of $\sigma$. To express this problem more succinctly, we introduce the additional notation

$$
F_{0}=\left[\begin{array}{cccccccc}
A & B & 0 & 0 & \cdots & 0 & 0 & b \\
-I & K & 0 & 0 & \cdots & 0 & 0 & 0 \\
0 & 0 & A & B & \cdots & 0 & 0 & 0 \\
0 & E_{1} & -I & K & \cdots & 0 & 0 & 0 \\
\vdots & \vdots & \vdots & \vdots & \ddots & \vdots & \vdots & \vdots \\
0 & 0 & 0 & 0 & \cdots & A & B & 0 \\
0 & E_{P} & 0 & 0 & \cdots & -I & K & 0 \\
0 & d_{1} & 0 & d_{0} & \cdots & 0 & 0 & 0 \\
\vdots & \vdots & \vdots & \vdots & \ddots & \vdots & \vdots & \vdots \\
0 & d_{P} & 0 & 0 & \cdots & 0 & d_{0} & 0
\end{array}\right]
$$

and for $k=1,2, \ldots, M$

$$
F_{k}=\left[\begin{array}{cccccccc}
0 & 0 & 0 & 0 & \cdots & 0 & 0 & 0 \\
0 & E_{k} & 0 & 0 & \cdots & 0 & 0 & 0 \\
0 & 0 & 0 & 0 & \cdots & 0 & 0 & 0 \\
0 & 0 & 0 & E_{k} & \cdots & 0 & 0 & 0 \\
\vdots & \vdots & \vdots & \vdots & \ddots & \vdots & \vdots & \vdots \\
0 & 0 & 0 & 0 & \cdots & 0 & 0 & 0 \\
0 & 0 & 0 & 0 & \cdots & 0 & E_{k} & 0 \\
0 & 0 & 0 & d_{k} & \cdots & 0 & 0 & 0 \\
\vdots & \vdots & \vdots & \vdots & \ddots & \vdots & \vdots & \vdots \\
0 & 0 & 0 & 0 & \cdots & 0 & d_{k} & 0
\end{array}\right]
$$

These matrices have $(2 N+1)(M+1)-1$ rows and $2 N(M+1)+1$ columns. A stationary solution exists for the optimization problem if

$$
\operatorname{Rank}\left[F_{0}+\sum_{k=1}^{M} \sigma_{k} F_{k}\right]<2 N(M+1)
$$

This multiparameter eigenvalue problem is generally quite difficult to solve. The special case of single heat engine or heat pump is an important exception which, as shown below, yields the familiar generalized eigenvalue problem of linear algebra. The general case of $M>1$, however, appears to be a challenging problem in numerical algebraic geometry.

\section{B. Generalized Eigenvalue Problem}

The special case $M=1$ corresponds to a single heat engine or heat pump. In the case, the rank condition boils down to

$$
\operatorname{Rank}\left[F_{0}+\sigma F_{1}\right]<4 N
$$

where $F_{0}$ and $F_{1}$ are $4 N \times 4 N$. Thus we obtain the generalized eigenvalue problem

$$
\operatorname{det}\left[F_{0}+\sigma F_{1}\right]=0
$$

The solution to the eigenvalue problem yields the optimal operating point.

\section{BILINEAR OPTIMIZATION}

There are several approaches one could use to obtain the optimization of a bilinear objective. In this paper, following the pioneering work of McCormack and others [10], [11], [13], we use a relaxation of the bilinear objective and constraints coupled with a branch-and-bound strategy to find a global minimum.

\section{A. Linearization and Relaxation}

By using an appropriate substitution into the bilinear problem, the bilinear objective and constraints are transformed into a linear optimization problem. The new variable is assumed to be linearly independent from the other variables, leading to a relaxation of the original problem [10], [11]. In this problem, the substitution we define is:

$$
z=T \sigma^{\top}
$$

with dimensions of $N \times M$. This leads to:

$$
f(T, \sigma, z)=R^{\top}\left(K T+\sum_{k} E_{k} z_{k}\right)-S^{\top}\left(\sum_{k} W_{k} z_{k}\right)
$$

The relaxed optimization problem with original constraints and additional substitution constraint are given by:

$$
\begin{aligned}
& \min _{t, \sigma, z} \quad f=R^{\top} q-S^{\top} w \\
& \text { s.t. } \quad q=K T+\sum_{k} E_{k} z_{k} \\
& w=\sum_{k} W_{k} z_{k} \\
& T^{L} \leq T \leq T^{U} \\
& \sigma^{L} \leq \sigma \leq \sigma^{U} \\
& \begin{aligned}
q^{L} & \leq q \leq q^{U} \\
w^{L} & \leq w \leq w^{U}
\end{aligned} \\
& z=T \sigma^{\top}
\end{aligned}
$$

Introducing the relaxation requirement that $z$ be linearly independent of $T$ and $\sigma$, four additional constraints replace Eq. 16e. Two constraints are a result of the convex underestimate and two are a result of the concave overestimate of the actual bilinear function. These are given by:

$$
\begin{aligned}
& z \geq T\left(\sigma^{U}\right)^{\top}+T^{U} \sigma^{\top}-T^{U}\left(\sigma^{U}\right)^{\top} \\
& z \geq T\left(\sigma^{L}\right)^{\top}+T^{L} \sigma^{\top}-T^{L}\left(\sigma^{L}\right)^{\top} \\
& z \leq T\left(\sigma^{U}\right)^{\top}+T^{L} \sigma^{\top}-T^{L}\left(\sigma_{U}\right)^{\top} \\
& z \leq T\left(\sigma^{L}\right)^{\top}+T^{U} \sigma^{\top}-T^{U}\left(\sigma^{L}\right)^{\top}
\end{aligned}
$$




\section{B. Branch and Bound Strategy}

Let $\mathcal{A}_{i}$ be the set of active branch and bound nodes on interval $i$. Additionally, let $j$ be an index containing all branch and bound nodes created, both open and closed. The following steps are executed to solve the relaxed optimization using a branch and bound technique. Unless otherwise stated, references to nodes refer to branch and bound nodes:

1) Calculate maximum possible entropy flux (Eq. 18). The minimum possible entropy flux for each engine is by definition zero $\left(\sigma^{L}=0\right)$.

$$
\sigma_{k, \circ}^{U}=\frac{\max \left[\operatorname{abs}\left(q^{U}\right) ; \operatorname{abs}\left(q^{L}\right)\right]}{\min \left(T^{L}\right)}, k=1, \ldots, M
$$

2) Initialize the upper and lower bounds on $f$ by finding the minimum and maximum values of the relaxed constrained problem, given above in Eq. 16a-16e and Eq. 17. This also tests the feasibility of the defined problem.

3) Trim the search region of the optimization by finding the minimum and maximum values of $T$ and $\sigma$ given the bounds on the function value (similar to step 8).

4) All open nodes are scanned. The first node for each of the following criteria is identified:

a) The lowest lower bound on $f$.

b) The lowest upper bound on $f$.

c) A maximum number of entropy fluxes on the lower edge.

d) A maximum number of entropy fluxes on the upper edge.

5) Each identified node (parent node) is branched by splitting the largest entropy range of the node in half to create two new nodes. The parent node is removed from and the new nodes are added to the set of open nodes $\mathcal{A}_{i}$. This step can create as few as 2 new nodes and as many as 8 new nodes (the same node can satisfy multiple criteria in step 4).

6) Upper and lower bounds on $f$ are computed for all new nodes using the relaxed optimization problem given in Eq. 16a-16e and Eq. 17.

7) Any open node whose lower bound of the objective function is higher than the lowest upper bound has been fathomed and may be eliminated:

8) Update absolute upper and lower bounds on entropy and temperature (Eq. 19) using the absolute upper and lower bounds on $f$ as constraints (Eq. 20):

$$
\begin{array}{cc}
\min _{T, \sigma, z} & T_{n}^{L} \text { s.t. } T_{n}^{L} \leq T_{n} \leq T_{n}^{U}, n=1, \ldots, N \\
\max _{T, \sigma, z} & T_{n}^{U} \text { s.t. } T_{n}^{L} \leq T_{n} \leq T_{n}^{U}, n=1, \ldots, N \\
\min _{T, \sigma, z} & \sigma_{k}^{L} \text { s.t. } \sigma_{k}^{L} \leq \sigma_{m} \leq \sigma_{k}^{U}, k=1, \ldots, M \\
\max _{T, \sigma, z} & \sigma_{k}^{U} \text { s.t. } \sigma_{k}^{L} \leq \sigma_{k} \leq \sigma_{k}^{U}, k=1, \ldots, M \\
& \\
& f_{\text {abs }}^{L} \leq f \leq f_{\text {abs }}^{U}
\end{array}
$$

9) Eliminate and trim current open nodes based on $\sigma$ values:

a) Eliminate nodes where the upper bound on $\sigma$ is less than the absolute lower bound.

b) Eliminate nodes where the lower bound on $\sigma$ is higher than the absolute upper bound.

c) Update the lower bound on $\sigma$ in nodes where the entropy range contains the absolute lower bound.

d) Update the upper bound on $\sigma$ in nodes where the entropy ranged contains the absolute upper bound.

10) Repeat from step 4 until the largest difference between the function values of open nodes and the current lowest upper bound are below some tolerance.

$$
\begin{cases}f_{\text {abs }}^{U}-\min \left(f_{j}\right)>f_{\mathrm{tol}}, \forall j \in \mathcal{A}_{i} & \text { repeat } \\ \text { otherwise } & \text { end }\end{cases}
$$

\section{EXAMPLES}

\section{A. Simple 1-Engine Model}

Fig. 5 gives an example of a simple 1-engine model. The following conditions are defined for the heat nodes:

\begin{tabular}{|c|c|c|c|c|c|}
\hline Var. & Min. & Max. & Var. & Min. & Max. \\
\hline \hline$T_{1}$ & 573.15 & 573.15 & $Q_{1}$ & 0 & 1500 \\
\hline$T_{2}$ & 277.6 & 573.15 & $Q_{2}$ & 0 & 0 \\
\hline$T_{3}$ & 277.6 & 573.15 & $Q_{3}$ & 0 & 0 \\
\hline$T_{4}$ & 277.6 & 277.6 & $Q_{4}$ & -1500 & 0 \\
\hline
\end{tabular}

Note that negative values of heat flux $\left(Q_{n}, n \in N\right)$ corresponds to heat being rejected.

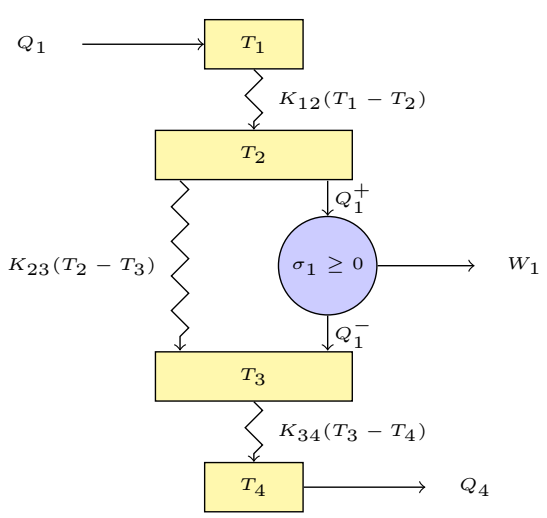

Fig. 5. An energy conversion network with one engine

The three conductance links in this model have parameter values $K_{12}=K_{34}=15$ and $K_{23}=1$. The engine is defined to have an isentropic efficiency of $0.95\left(\eta_{1}=0.95\right)$ and bounds on the work are $0 \leq W_{1} \leq 1500$. The price of $Q_{1}$ is $\$ 0.10$ per unit, and the cost of disposal of waste heat $Q_{4}$ is $\$ 0.01$ per unit. The value of the work produced $W_{1}$ is $\$ 1$ per unit.

This model was simulated using the methodology outlined in section V-B. The simulation calculated a function value 
of $\$ 186.22$ profit and values for the adiabatic heat nodes of $T_{2}=490.6$ and $T=338.9$. This example is also quite easy to analyze by varying the entropy flux and solving the remaining linear equations directly. This approach produced the same results as the simulation. Figure 6 shows the plot of profit versus work output as well as minimum cost (red square) and maximum work output (black circle). Note that maximizing work output does not maximize profit in this case.

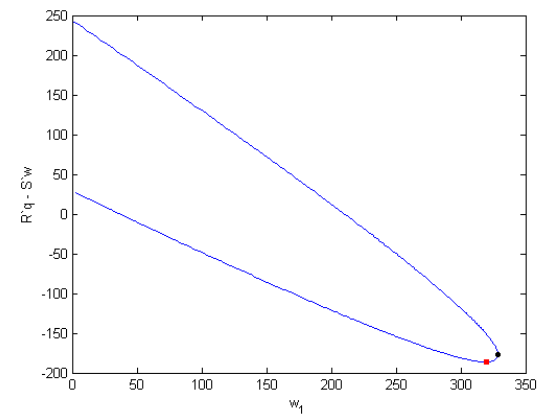

Fig. 6. Plot of profit versus work for a single engine ECN.

\section{B. Geothermal Air-Conditioning}

Fig. 7 shows a geothermal air-conditioning model based on a paper by Davis and $\mathrm{Wu}$ [5]. The following temperature and heat flux conditions are defined:

\begin{tabular}{|c|c|c|c|c|c|}
\hline Var. & Min. & Max. & Var. & Min. & Max. \\
\hline \hline$T_{1}$ & 375 & 375 & $Q_{1}$ & 0 & 250,000 \\
\hline$T_{2}$ & 307 & 375 & $Q_{2}$ & 0 & 0 \\
\hline$T_{3}$ & 307 & 375 & $Q_{3}$ & 0 & 0 \\
\hline$T_{4}$ & 307 & 307 & $Q_{4}$ & $-250,000$ & 0 \\
\hline$T_{5}$ & 290 & 290 & $Q_{5}$ & 0 & 250,000 \\
\hline$T_{6}$ & 190 & 290 & $Q_{6}$ & 0 & 0 \\
\hline$T_{7}$ & 290 & 390 & $Q_{7}$ & 0 & 0 \\
\hline
\end{tabular}

The conductance links in this model, as defined in the Davis and $\mathrm{Wu}$ paper, have the values $K_{12}=K_{34}=$ $1000 \mathrm{~W} / \mathrm{K}$ and $K_{56}=K_{74}=5000 \mathrm{~W} / \mathrm{K}$. The isentropic efficiency of both engines are $1\left(\eta_{1}=1, \eta_{2}=1\right)$. The bounds on work output are $0 \leq W_{1} \leq 50000$ and $-50000 \leq W_{2} \leq$ 0 .

Davis and $\mathrm{Wu}$ reported the maximum cooling capacity $\left(Q_{5}\right)$ was $11.4 \mathrm{~kW}$. Davis and $\mathrm{Wu}$ also reported the optimal operating temperatures for non-isothermal nodes from their simulation as $T_{2}=357, T_{3}=323, T_{6}=286$, and $T_{7}=311$.

We aimed to test their results with the modeling and optimization method outlined in this paper. To calculate the maximum cooling capacity (or minimum negative of cooling capacity) $Q_{5}$ was given a value of $-1\left(r_{5}\right)$ with all other $r_{i}, i \in N$ set to zero and $S$ set to a zero vector of length $M+P$.

The simulation found the maximum cooling capacity to be $19.4 \mathrm{~kW}$ with the optimal operating temperatures in agreement with Davis and $\mathrm{Wu}$. This cooling capacity and these

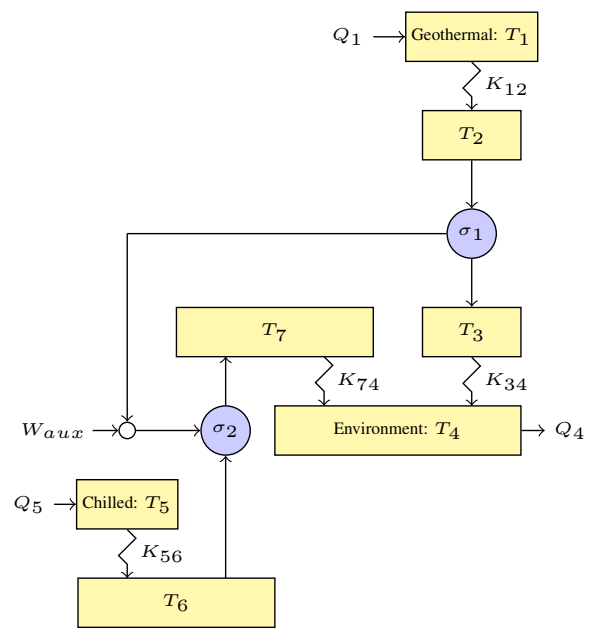

Fig. 7. A geothermal model

operating temperature are consistent with the underlying thermodynamic formulas involved.

\section{CONCLUSIONS}

Our analysis of energy conversion networks introduces a class of bilinear models for the optimization and risk management. In addition to a number of theoretical results, a branch and bound methodology was introduced for the linearized and relaxed form of the bilinear objective function. Subsequent research will demonstrate the utility of these models for the stated objectives.

\section{REFERENCES}

[1] Bjarne Andresen, Peter Salamon, and R. Stephen Berry. Thermodynamics in finite time. Physics Today, 37(9):62-70, September 1984.

[2] Anees Attarwala and Jeffrey C. Kantor. On the integration of finance and process operations. In AIChE 2007 Annual Meeting, 2007.

[3] Herbert B. Callen. Thermodynamics and an Introduction to Thermostatics. John Wiley \& Sons, second edition, 1985.

[4] Lingen Chen, Chih Wu, and Fengrui Sun. Finite time thermodynamic optimization or entropy generation minimization of energy systems. Journal of Non-Equilibrium Thermodynamics, 24:327-359, 1999.

[5] Gregory W. Davis and Chih Wu. Finite time analysis of a geothermal heat engine driven air conditioning system. Energy Conversion and Management, 38(3):263-268, February 1997.

[6] S.J. Deng and S.S. Oren. Electricity derivatives and risk management. Energy, 31(6-7):940-953, 2006.

[7] Alexander Eydeland and Krzysztof Wolyniec. Energy and Power Risk Management: New Developments in Modeling, Pricing and Hedging. Wiley, 2003.

[8] Kendell R. Jillson and B. Erik Ydstie. Process networks with decentralized inventory and flow control. Journal of Process Control, 17(5):399-413, June 2007.

[9] Jeffrey Kantor and Anees Attarwala. Modeling, analysis, and optimization of complex energy utilities. In ADCONIP, 2008.

[10] Sangbum Lee and Ignacio E. Grossmann. Global optimization of nonlinear generalized disjunctive programming with bilinear equality constraints: applications to process networks. Computers \& Chemical Engineering, 27(11):1557-1575, November 2003.

[11] Garth P. McCormick. Computability of global solutions to factorable nonconvex programs: Part i - convex underestimating problems. Mathematical Programming, 10(1):147-175, December 1976.

[12] Dragana Pilipovic. Energy Risk: Valuing and Managing Energy Derivatives. McGraw-Hill, second edition edition, 2007.

[13] Hanif D. Sherali and Amine Alameddine. A new reformulationlinearization technique for bilinear programming problems. Journal of Global Optimization, 2(4):379-410, December 1992. 